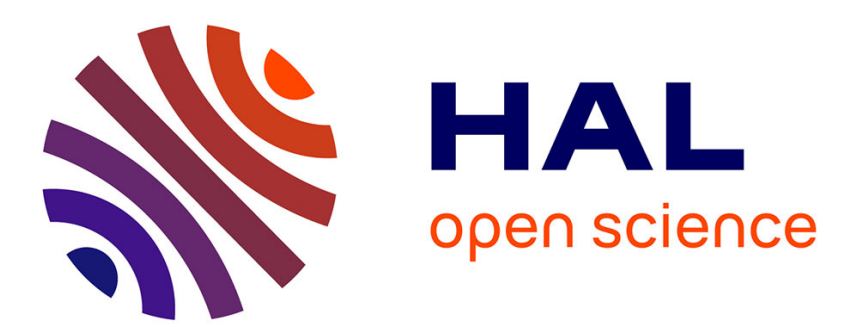

\title{
ESco: Eligibility Score-based Strategy for Sensors Selection in CR-IoT: Application to LoRaWAN
}

Maxime Mroue, Abbass Nasser, Benoît Parrein, Ali Mansour, Chamseddine Zaki, Eduardo Motta Cruz

\section{- To cite this version:}

Maxime Mroue, Abbass Nasser, Benoît Parrein, Ali Mansour, Chamseddine Zaki, et al.. ESco: Eligibility Score-based Strategy for Sensors Selection in CR-IoT: Application to LoRaWAN. Internet of Things, 2021, 13, pp.100362. 10.1016/j.iot.2021.100362 . hal-03120664v2

\section{HAL Id: hal-03120664 \\ https://hal.science/hal-03120664v2}

Submitted on 14 Oct 2021

HAL is a multi-disciplinary open access archive for the deposit and dissemination of scientific research documents, whether they are published or not. The documents may come from teaching and research institutions in France or abroad, or from public or private research centers.
L'archive ouverte pluridisciplinaire HAL, est destinée au dépôt et à la diffusion de documents scientifiques de niveau recherche, publiés ou non, émanant des établissements d'enseignement et de recherche français ou étrangers, des laboratoires publics ou privés. 


\title{
ESco: Eligibility Score-based Strategy for Sensors Selection in CR-IoT: Application to LoRaWAN
}

\author{
Maxime Mroue $^{a *}$, Abbass Nasser ${ }^{b, c}$ Benoît Parrein $^{d}$, Ali Mansour ${ }^{c}$, \\ Chamseddine Zaki ${ }^{e}$, Eduardo Motta $\mathrm{Cruz}^{a}$ \\ ${ }^{a}$ Université de Nantes, CNRS, IETR UMR 6164, F-85000 La Roche sur Yon, France \\ ${ }^{b} I C C S-L a b$, Computer Science Department, AUCE, Beirut, Lebanon \\ ${ }^{c}$ LABSTICC, UMR CNRS 6285, ENSTA Bretagne, 2 Rue François Verny, 29806 Brest, \\ France \\ ${ }^{d}$ Université de Nantes, CNRS, LS2N UMR 6004, F-44000 Nantes, France \\ ${ }^{e}$ MIS Department, Lebanese University, Rachaya, Lebanon
}

\begin{abstract}
In this paper, a battery-powered Internet of Things assisting Cognitive Radio (CR-IoT) network is considered. In CR-IoT network, a set of IoT End Nodes (ENs) are assumed to cooperatively sense the channel. Each EN performs a Spectrum Sensing (SS) and reports its decision to a Fusion Center in order to reach the decision on the PU status. Such monitoring of PU comes at the cost of additional energy resources consumed by the EN to perform SS and reporting, and raises the need to set a suitable protocol to manage the data exchange. The extra operations may exhaust the energy of the EN battery, especially when Low Power Wide Area Networks (LPWAN) technology is used. In our manuscript, an Eligibility Score-based (ESco) Strategy is defined to reflect the eligibility of an EN to make a reliable SS taking into consideration its battery level. Our strategy is adapted to LoRaWAN Class-B protocol in order to handle real world scenarios. Necessary modifications are introduced to the existing protocol in order to support the synchronization between the ENs and the Application Server, (at which the SS need is identified), and the on-demand nature of the SS requests. To evaluate our strategy, LoRa sensors' parameters are considered in our simulations. The numerical results highlight the efficiency of the proposed strategy by showing the extension of the ENs' lifetime compared to classical strategies.
\end{abstract}

Keywords: Internet of Things, Network Lifetime, Cognitive Radio, Spectrum Sensing, LoRa Networks, LoRaWAN Protocol.

${ }^{*}$ Corresponding author. Email: maxime.mroue@univ-nantes.fr 


\section{Introduction}

According to [1, 75 billions of Internet of Things (IoT) devices will be connected to the Internet by 2025 . IoT will cover almost all the society life sectors, such as: urban, industry, medicine, transportation, security surveillance, etc

5 2, 3. The expected huge number of devices will require special attention to the physical layer in order to maintain the service quality [4, 5].

In turn, Cognitive Radio (CR) is one of the most studied wireless technologies in the last two decades [6, 7, 8]. CR was introduced to mitigate the 10 spectrum scarcity problem due to the ever increasing demand on the wireless communications. The $\mathrm{CR}$ system is based on sharing the spectrum between primary (PU) and secondary (SU) users. A PU has the exclusive right to access its spectrum band at any time, while SU, as an opportunistic user, can only access the spectrum whenever $\mathrm{PU}$ is absent. If PU resumes its transmission while $\mathrm{SU}$

15 is active, then SU should immediately vacate the channel. The decision on the PU channel availability is the mission of the Spectrum Sensing (SS) part of CR.

To tackle the needs of the additional frequency resources, the shifting to the CR-based communication requires SS to keep aware of the PU activity. Due 20 to the deployment of IoT networks, their sensors may successfully perform the SS operation. Thus, the monitoring of the primary channel can be exploited according to two scenarios:

- CR-based Communication for IoT: In this scenario, IoT network performs SS for its interest due to its need for additional spectrum resources to send extra data 9. This scenario may happen when the number of nodes in the IoT network is largely increased, which may cause high pressure on the frequency resources and require additional spectral bands. In this scenario, the EN may perform SS in addition to its initial task in sensing physical measures, such as: the temperature, the humidity, etc.

- Providing Spectrum Sensing as a Service (SSaaS): the CR-IoT may deliver a service of SS for another network. Hence, Sensing as a Service strategy is applied [10, 11]. In this case, CR-IoT receives a request from another network/operator asking about the status of a given channel within a geographical area. Accordingly, the CR-IoT asks the ENs to perform SS and report their decisions on the availability of a given frequency band for the sake of another network/operator. This second scenario is particularly covered in this paper.

\subsection{CR-based Communication for IoT}

In this section, an overview on related works to the CR-based communication for IoT is presented. Due to the huge expected number of IoT devices, CR 
has been proposed as a major solution to tackle the problem of the scarcity of frequency resources on the physical layer 4 4. CR considers the IoT nodes as 45 secondaries and provides them with a high flexibility since they may access any frequency band whenever PU is absent.

The scarcity of the spectrum and the static allocation policy are presented as main reasons to adopt the CR-based IoT in future radio technology generations [4. The latter reference details the implementation of CR in IoT network in 50 addition to the standardization and the applications of the CR-based IoT.

Energy-Throughput trade-off of wireless sensor network is studied in [12] in the context of cooperative SS and dedicated to low-power devices. The main contribution of that study focuses on minimizing the consumed energy while satisfying the requirements of secondary throughput and the primary signal detection.

55 In [13, CR is extended to IoT networks in order to make a self-reconfigurable, interference-free, and intelligent IoT networks. CR xan be helpful to Machineto-Machine communications as suggested by 14, 15.

A dynamic spectrum allocation for IoT is proposed in [16, where a cognitive radio-based strategy is applied in order to avoid the interference among the IoT

${ }_{60}$ nodes as SU and PU. To achieve such a goal, the proposed strategy aims to work in both licensed and unlicensed bands in a dynamic manner.

Last but not least, cognitive radio framework is proposed in [17] for IoT networks. The multiband cooperative SS is applied on IoT nodes, assumed to be environment-aware and self-reconfigurable, in order to reduce the energy con-

65 sumption at every node. Multiband SS is adopted due to two main reasons: 1) the need of the IoT networks to cover several frequency channels to fulfil their transmission requirement, and 2) having several available frequency channels makes the frequency handoff simpler and faster.

\subsection{Spectrum Sensing as a Service}

The SSaaS is inspired by the concept of Sensing as a Service which can be adopted by a Wireless Sensor Network or IoT network to deliver a service to another network or operator. Data like humidity, temperature, pressure, etc. can be monitored by a WSN/IoT within a geographical area and provided to

75 another operator. Similarly to the concept of Sensing as a Service, SSaaS has been proposed to provide another network/operator by the channel availability within a geographical area. The authors of 18 proposed the separation of the spectrum sensing task from the other tasks of a cognitive radio, by using the dedicated infrastructure of a WSN/IoT. In this case, the WSN/IoT outcomes

so a list of available channels in response to a request of a Cognitive Radio-based network.

The SSaaS imposes several challenges related to the business model to be adopted between the WSN/IoT and the operator to be provided. Other challenges related to the nodes selection and the energy efficiency have been studied

${ }_{85}$ in the literature in the sake of providing reliable and cost-effective service of SS [10, 11, 18, 19, .

Our manuscript mainly focuses on this scenario, where we aim at analysing the 
selection strategy of the ENs of the IoT network in production, and adapt the suitable protocol to exchange the data between the ENs and the upper entity of the IoT network.

One of the main challenging issues when assigning the SS task to an IoT network, in both scenarios detailed above, is the energy autonomy which is related to the battery lifetime 20]. For instance, in Low Power Wide Area Network 5 years 20 . This is related to the battery echnology and the transmion ifications of the IoT networks, especially the small duty cycle of less than $1 \%$ as in LoRa network 21. The sensing operation required by the cognitive system reduces the IoT node's life due to the additional energy resources required by the SS function, where two major additional power consuming operations have to be done:

1. Making a decision on the channel by applying a sensing technique.

2. Reporting the decision to the Fusion Center (FC). This operation may rapidly consume the battery energy of the sensor due to the additional transmission operation.

However, the sensing of a given channel is the mission of several ENs in CRIoT networks. This will positively impact both the energy efficiency and the performance since the consumed energy per node will reduced and the spatial diversity will improve the sensing outcome 22. Each EN makes a decision on the channel and sends it to the FC, which combines all decisions on that channel to output a final decision. Thus, the selection of the ENs performing the SS becomes an essential challenge of the FC. From one hand, selecting these ENs may be a function of the required number of ENs to perform the SS in order to fulfil the detection requirement (target false alarm and detection probabilities).

115 On the other hand, this selection should take into consideration the battery level of the EN and its detection reliability, which is related to Signal to Noise Ratio (SNR) observed at that node. If an EN is about to be exhausted, it is recommended not to ask it to perform further SS. In addition, when the EN's SNR is bad, EN should treat a high number of received samples to outcome a 120 reliable decision 23. This fact may degrade the EN's battery. However, the ENs selected to perform the SS may vary from one SS operation to another. This fact will ensure a high participation of the node in the SSaaS task and to decrease the number of transmission of each node so it respects the LoRaWAN specifications related to the duty cycle.

In this paper, an Eligibility Score-based (ESco) strategy for IoT network providing SSaaS is proposed, where the selection of IoT ENs respects both the SS requirements and the energy level of the ENs. LoRaWAN is taken as case study to investigate the performance of our strategy, where we adapted the 130 new strategy requirements to the LoRaWAN protocol. LoRaWAN is basically designed for uplink operations in which the ENs report the data to the Application Server (AS) after passing by the Gateway (GW) and the Network Server 


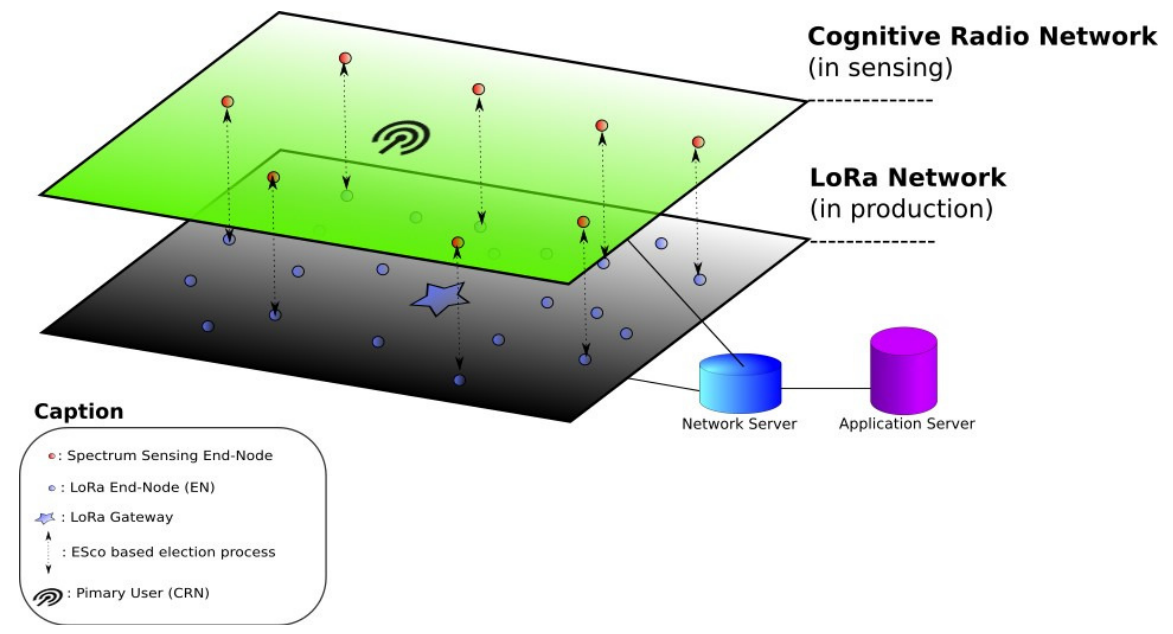

Figure 1: General view of the network topology for the proposed ESco solution: the elected End-Nodes from a standard LoRa network carry on the Spectrum Sensing for the Cognitive Radio Network layer

(NS) sparsely in time with a relatively low throughput. This fact ensures a long lifetime to the ENs measured in years instead of hours or months. In our appli135 cation, the need for spectrum resources is identified at the AS, which requests the ENs to collect sensing data on a given channel. Then, the ENs should report their SS decisions on such channel to the AS, which plays the role of the FC in our work, upon request. Hence, we are in on-demand scenario [24, which is not fulfilled in existing LoRaWAN protocol. Therefore, we are suggesting to adapt the protocol to better fit our application. Figure 1 gives an overview of the adopted system model. The whole network is under the control of the NS and AS. NS manages the transmission parameters of the LoRa ENs such the Spreading Factor (SF) and the operating frequency. However, being the only entity in the LoRa network allowed to read the sensing content of the EN's message, AS elects the most eligible nodes to perform the SS using ESco strategy based on the data received from these nodes. A selected node participates in the CR operations and works at the CR network level to sense the PU activity. The main contributions of this manuscript can be summarized as follows:

1. Based on an evaluated Eligibility Score (ES) defining the eligibility of each EN to perform the SS, a new strategy called ESco of ENs selection is proposed. ESco ensures that the CR-IoT meets the detection requirements to achieve a high spectrum efficiency and a good protection of PU against the ENs transmission interference. In addition to the SS reliability, the 

where we derive the to establish the SS. Section 4 describes the adopted protocol to exchange the data between the ENs and the AS. LoRaWAN-based protocol is presented. Numerical simulations are presented in section 5 , where we evaluate our proposed throughput. Section 6 concludes our work.

Table 1: List of Abbreviations and Symbols

\begin{tabular}{ll}
\hline Abbreviation/Symbol & Definition \\
\hline AS & Application Server \\
BS & Base Station \\
BW & Bandwidth \\
CR-IoT & Cognitive Radio-based Internet of Things \\
CR & Cognitive Radio \\
EN & End-Node \\
ES & Eligibility Score \\
FC & Fusion Center \\
GW & Gateway \\
IoT & Internet of Things \\
IP & Internet Protocol \\
LoRa & Long Range \\
LoRaWAN & LoRa Wide Area Network \\
LPWAN & Low-Power Wide Area Network \\
NS & Network Server \\
PL & PayLoad \\
PU & Primary User \\
SF & Spreading Factor
\end{tabular}




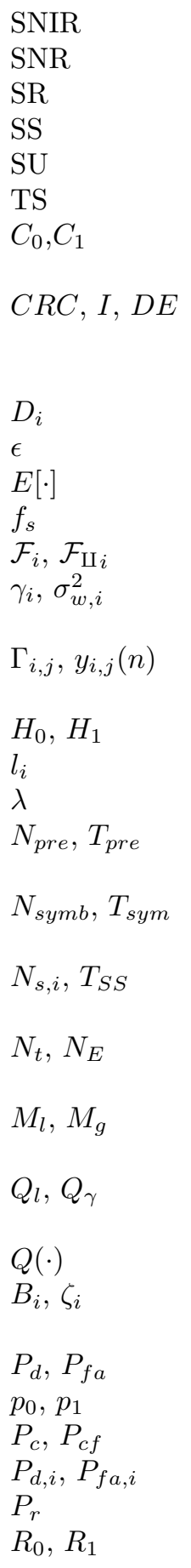

Signal to Noise and Interference Ratio

Signal to Noise Ratio

Sensing-Reporting

Spectrum Sensing

Secondary User

Test Statistic

The channel capacity of the secondary transmission when PU is absent and active respectively

They stand respectively for: the presence or not of the CRC field in the physical message, the coding rate, and the low data rate optimization enabling.

The decision of the spectrum sensing made by the $\mathrm{EN}_{i}$

Quantization error

Mathematical expectation

Sampling frequency

The ES function of the $\mathrm{EN}_{i}$ and its quantization respectively

The SNR of the PU signal and the variance of the noise observed at $\mathrm{EN}_{i}$

The test statistic and the received signal of the channel $j$ at the $\mathrm{EN}_{i}$

Hypotheses that $\mathrm{PU}$ is absent or active respectively

Battery level of $\mathrm{EN}_{i}$

Threshold of comparison in spectrum sensing

Number of synbols in the Preamble message and its time respectively.

The number of transmitted symbols in a packet and their duration respectively.

The number of samples and the needed time respectively needed by the $\mathrm{EN}_{i}$ in $\mathrm{SS}$.

Total number of ENs and number of End-Nodes selected to make spectrum sensing

The bits number reserved to represent the battery levels and the SNR respectively

Set of quantization levels of the battery levels and the SNR respectively

Gaussian Q-Function

The $i$ th level of quantization $\mathrm{f}$ the battery levels and the SNR respectively

Probability of detection and false alarm respectively

Probability that PU is active or absent respectively.

Probability of collision and collision-free respectively

Probability of detection and false alarm respectively of the $\mathrm{EN}_{i}$

Probability that PU returns active

The useful and the colliding throughputs of the secondary transmission respectively 


\begin{tabular}{ll}
$R x 1, R x 2$ & First and second receiving windows respectively \\
$s_{i, j}(n), w_{i, j}(n)$ & The PU received signal and the noise at the channel $j$ at the $\mathrm{EN}_{i}$ \\
$T_{b c n}$ & Beacon's period \\
$T_{T x}$ & Duration of the transmission time \\
$T x$ & Transmission window in LoRaWAN protocol \\
$U_{i}$ & The $i$ th user selected to perform the spectrum sensing \\
$W$ & Bandwidth of the secondary transmission \\
$\bar{x}$ & The target value of $x$ \\
\hline
\end{tabular}

\section{System Model}

The considered CR-IoT consists of a certain number of ENs, with $N_{t}$ ENs are able to make the SS. The $N_{t}$ nodes serve as spectrum sensors by reporting their measured decisions to the FC (Application Server), which makes the final decision. Note that the channel to be sensed is different from the actual channel used by the AS and ENs to exchange the data through the GW. To reach this goal, the nodes are assumed to be equipped with a cognitive radio module allowing them to configure their frequency parameters to observe a certain channel as per the request of AS.

In order to exploit the spatial diversity of the $N_{t}$ ENs, cooperative SS is used. These $N_{t}$ nodes, or some of them, must cooperatively report their decisions on a given channel to the AS via the GW and the NS. In turn, AS considers the decisions of the ENs to reach a final decision on that channel.

In our scenario, the CR-IoT networks may sense a set of $N_{c}$ channels. The sensing operation of each channel is the task of $N_{E} \leq N_{t}$ ENs, where each of them evaluates a Test Statistic (TS). The $i$ th EN (denoted by $\mathrm{EN}_{i}$ ), $i \in\left[1, N_{E}\right]$, should discriminate between two hypotheses $H_{0}$ : PU is idle and $H_{1}$ : $\mathrm{PU}$ is active:

$$
\begin{aligned}
& y_{i, j}(n)=w_{i, j}(n) \text { under } H_{0} \\
& y_{i, j}(n)=s_{i, j}(n)+w_{i, j}(n) \text { under } H_{1}
\end{aligned}
$$

where $y_{i, j}(n), s_{i, j}(n)$, and $w_{i, j}(n)$ are respectively the received signal, the received PU signal including the channel effect, and the noise observed at the $i$ th EN at the channel $j \in\left[1, N_{c}\right]$.

In this paper, the widely used non-coherent Energy Detector (ED) is adopted 25. ED consists in evaluating the TS $\Gamma_{i, j}$ on the received signal $y_{i, j}(n)$ at the channel $j \in\left[1, N_{c}\right]$ and compare it to a predefined threshold $\lambda$ to make a binary decision: 1 if $\mathrm{PU}$ is detected as active, and 0 if $\mathrm{PU}$ is diagnosed absent.

$$
\Gamma_{i, j}=\frac{1}{N_{s, i}} \sum_{n=1}^{N_{s, i}}\left|y_{i, j}(n)\right|^{2} \underset{0}{\gtrless} \lambda
$$

where $N_{s, i}$ is the number of received samples at the $i$ th EN used to evaluate $\Gamma_{i, j}$. 
The ENs should inform the AS by their decisions. Thus, the decision $D_{i}$ of $\mathrm{EN}_{i}$ should be reported using a control channel to the AS in order to make a final decision [22, 26]. In our work, the "Or" logic rule, i.e. the minimum risk strategy, is applied on the received decisions of the ENs. The overall probability of detection $P_{d}$ and false alarm $P_{f a}$ can be evaluated as follows [22]:

$$
\begin{gathered}
P_{d}=1-\prod_{i=1}^{N_{E}}\left(1-P_{d, i}\right) \\
P_{f a}=1-\prod_{i=1}^{N_{E}}\left(1-P_{f a, i}\right)
\end{gathered}
$$

where $P_{d, i}$ and $P_{f a, i}$ are the probability of detection and the probability of false alarm at $i$ th EN respectively.

Assuming that the received signal samples are independent and identically distributed (i.i.d.), thus $P_{d, i}$ and $P_{f a, i}$ are presented as follows [27]:

$$
\begin{array}{r}
P_{d, i}=Q\left(\frac{\sqrt{N_{s, i}}\left(\frac{\lambda}{\sigma_{w, i}^{2}}-\gamma_{i}-1\right)}{\gamma_{i}+1}\right) \\
P_{f a, i}=Q\left(\sqrt{N_{s, i}}\left(\frac{\lambda}{\sigma_{w, i}^{2}}-1\right)\right)
\end{array}
$$

where $Q(\cdot)^{1}$ is the Gaussian $Q$-function, and $\sigma_{w, i}^{2}$ and $\gamma_{i}$ are the variance of $w_{i, j}(n)$ and the observed $\mathrm{SNR}$ at the the $\mathrm{EN}_{i}$ respectively.

As it can be shown from previous equations, $N_{E}, N_{s, i}$ and $\gamma_{i}$ control the SS performance. However, for target $P_{d}$ and $P_{f a}, N_{s, i}$ and $N_{E}$ become functions of $\gamma_{i}$. More $\gamma_{i}$ increases more the required $N_{E}$ and $N_{s, i}$ decrease. In turn, high $N_{s, i}$ leads to a high consumption of the battery of the EN, and usually $N_{s, i}$ decreases with $N_{E}$. The optimal solution that takes both the SS performance and the power consumption into consideration is to fulfil the target $P_{d}$ and $P_{f a}$ with low $N_{s, i}$ and $N_{E}$. Thus, the FC should be able to select the best ENs to perform the SS taking into consideration the battery level of these ENs and their SNRs.

\section{Proposed ENs Selection Strategy}

In order to optimize the consumption of the energy related to SS among the network's ENs, we propose to evaluate an Eligibility Score $(\mathrm{ES}) \mathcal{F}_{i}\left(\gamma_{i}, l_{i}\right)$ of each EN, which takes into consideration the needed $N_{s, i}$ to fulfil the SS requirements at the $\mathrm{EN}_{i}$ and its battery level $l_{i}$. In fact, in the classical cooperative

${ }^{1} Q(x)=\frac{1}{\sqrt{2 \pi}} \int_{x}^{+\infty} \exp \left(-\frac{u^{2}}{2}\right) d u[28]$ 

Joule/Sample. Such ES indicates how much the EN could spend energy for one sample. Thus, more the ES increases more the EN proves itself to be eligible. In addition, in the equation (8), $N_{E}$ and $\gamma_{i}$ are represented by $N_{s, i}$, since the latter inversely changes with $N_{E}$ and $\gamma_{i}$ for target $P_{d}$ and $P_{f a}$. The optimal choice derived from equation (8) corresponds to a high $l_{i}$ with a low $N_{s, i}$.

\subsection{Setting of Sensing Parameters}

The main goal of the CR network is to reach a high spectrum efficiency while protecting as possible the PU from the secondary interference. This goal can

\footnotetext{
${ }^{2}$ In LoRa technology, several GWs may cooperatively localize an EN using a triangulation technique.
} 
be reformulated as follows:

$$
\begin{array}{r}
P_{d} \geq \overline{P_{d}} \\
P_{f a} \leq \overline{P_{f a}}
\end{array}
$$

where $\overline{P_{f a}}$ and $\overline{P_{d}}$ are the target false alarm rate and detection probability in the CR-IoT network respectively.

Assuming all the ENs involved in the SS have the same target $\overline{P_{d, i}}$ and $\overline{P_{f a, i}}$, then by satisfying equations (4) and (5) we obtain:

$$
\begin{gathered}
\overline{P_{d, i}}=1-\sqrt[N_{E}]{1-\overline{P_{d}}} \\
\overline{P_{f a, i}}=1-\sqrt[N]{1-\overline{P_{f a}}}
\end{gathered}
$$

Here, $N_{E}$ represents the number of ENs which have the highest $N_{E}$ ESs:

$$
\left[U_{1}, U_{2}, \ldots, U_{N_{E}}\right]=\max _{\mathcal{F}_{i}\left(\gamma_{i}, l_{i}\right)} \operatorname{EN}_{i}, i \in\left[1, N_{t}\right]
$$

where $U_{i}$ is the $i$ th user selected to perform the SS, and $N_{t}$ is the total number of ENs in CR-IoT network. These ENs must meet $\overline{P_{d, i}}$ and $\overline{P_{f a, i}}$. To do so, the EN should take into consideration the number of samples $N_{s, i}$ to adopt. Using equations (6) and (7), we can obtain:

$$
N_{s, i}=\left[\frac{\alpha_{i}-\beta_{i}}{\gamma_{i}}-\beta_{i}\right]^{2}
$$

where $\alpha_{i}=Q^{-1}\left(\overline{P_{f a, i}}\right)$ and $\beta_{i}=Q^{-1}\left(\overline{P_{d, i}}\right)$.

As per the equation above, $N_{s, i}$ and $\gamma_{i}$ are inversely proportional. Low SNR necessitates high $N_{s, i}$ to converge to an accurate decision of SS. This requires additional power resources. Thus, ENs with low $\gamma_{i}$ are dying faster than ENs with high SNR. However, this issue can be controlled by the ES: when the AS selects two ENs of the same ES to perform the SS, if the first EN is of higher $l_{i}$ than the other one, then it performs the SS with a higher $N_{s, i}$. In this case, the second EN uses certainly lower $N_{s, i}$, and subsequently it does not require 245 to spend a large amount of energy as such as the first one. Thus, the proposed ESco is dynamic and adaptable with respect to both needed number of samples and the available battery's energy.

\section{Application to LoRa Network}

LoRa (Long Range) is a spread spectrum modulation technique derived from

Chirp Spread Spectrum (CSS) technology. The Gateway (GW) coverage of LoRa network may achieve up to $20 \mathrm{Km}$ in rural areas and $5 \mathrm{~km}$ in urban areas. The data rate of LoRa network may vary from 0.3 to $50 \mathrm{kbps}$ depending on the Spreading Factor (SF), which varies between 7 and 12, and the adaptive data 

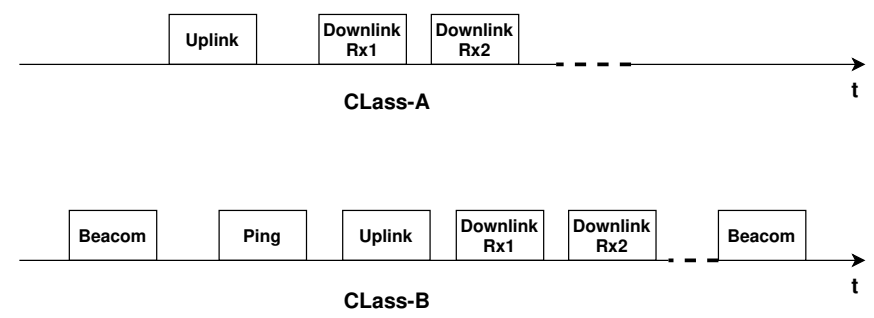

Figure 2: Time windows of LoRaWAN classes A and B: Class-B ensures a synchronization between FC and EN thanks to the Beacon.

rate algorithm. The SF selection has a great impact on the lifetime of the EN and may depend on several factors such as the distance from the EN to the GW, the network capacity, the packet error ratio, etc. [34, 35]. On the other hand, LoRaWAN defines the communication protocol of the LoRa technology. LoRaWAN networks are defined in a star topology, where the GW receives the data from the EN inside the network and forwards it to the NS and AS. The 260 communications between EN and GW are done using LoRa, while communications between GW and NS use Internet Protocol (IP)-based technologies such as $4 \mathrm{G}$ or WiFi. NS is considered as the manager of the LoRaWAN network, where it controls the selection of SF, the operating frequency, the network capacity, etc.. NS controls the network parameters while the Application Server (AS) is the only entity that could read the message content of the ENs. For that reason, AS could manage the proposed ESco strategy since the selection process is based on the data sent by the ENs. Thus, AS play the role of the FC of the ENs participating in the SS.

\subsection{Protocol of data exchange between FC and ENs}

As previously mentioned, AS plays the FC role in our network. Therefore, the need for spectral resources should be identified at the AS, which sends a request to the ENs via the NS and the GW respectively. The communication between GW and ENs is assumed to be established on a reserved control channel. The AS, playing the role of $\mathrm{FC}$, is assumed to be equipped with a database to 275 save data on the ENs especially $\mathcal{F}_{i}\left(l_{i}, N_{s, i}\right)$. Thus, the ENs, eligible to make the $\mathrm{SS}$, should be ready once the AS sends a request following on-demand fashion of the data exchange. This insists a synchronization between the AS and the ENs. As per LoRa regulations the GW broadcasts a Beacon each $128 \mathrm{sec}$ [36. Then, to respect this regulation, the ENs may report a decision on a channel every $128 \mathrm{sec}$ at least.

For LoRaWAN case study, we can distinguish two main classes: Class-A and Class-B 37, 38. In Class-A, no synchronization is made between GW and EN, thus, it becomes hard to apply in our scenario since the ENs should response once the AS requests. By contrast, in Class-B, the synchronization is ensured 285 thanks to a Beacon sent by the GW to the ENs. This Beacon is considered as a timing reference to the ENs, which can be used by the AS to initiate SS 


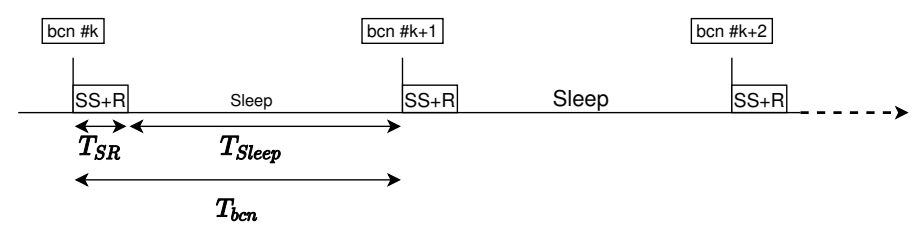

Figure 3: The behavior of the ENs during the SR operations: ENs wakes up for $T_{S R}$, then sleep till the next SR operation

operation. Then, the ENs can periodically open receive windows called "ping slots" to initiate an uplink operation $T x$ from the EN. Following each uplink transmission, the EN opens two short reception windows, $R x 1$ and $R x 2$, to receive data from the NS (see figure 2).

Upon the request of the AS, the $N_{E}$ ENs make SS and Reporting (SR) operations in $T x{ }^{3}$ The ENs participating in the sensing operation send their decisions in addition to the updated $l_{i}$ and $\gamma_{i} \cdot \gamma_{i}$ lets the AS calculates $N_{s, i}$ as per equation (14), and subsequently evaluate $\mathcal{F}\left(l_{i}, N_{s, i}\right)$. Evaluating $\mathcal{F}\left(l_{i}, N_{s, i}\right)$ 295 at the AS reduces the computational complexity at the EN and ensures that EN meets the detection requirement, i.e., $\overline{P_{d}}$ and $\overline{P_{f a}}$, since the SS requirements reflected by equation (14) could be changed from a primary channel to another according to the tolerable collision rate between PU and SU [39, 40]. Regarding the remaining ENs, which do not participate in SR, they remain silent but 300 receive from the AS through the NS and the GW.

In turn, after receiving the SS-related data in $T x$, AS makes the final decision on the PU channel and updates the database of the ENs based on the received data to select the most eligible $N_{E}$ to perform the SS in the next round. These $N_{E}$ ENs are selected based on their $\mathcal{F}_{i}\left(l_{i}, N_{s, i}\right)$. The $\mathrm{SR}$ operation is terminated 305 by informing the $N_{E}$ ENs of the next SR operation by their task, and providing them with $N_{s, i}$ to be used in the next round within $R x 1$ time slot. Indeed, other parameters are sent by the NS to the EN according to the classical LoRaWAN protocol such as the SF and the operating frequency to be adopted in the next transmission operation. Once SR operation is done, the ENs go to sleep mode with a time $T_{\text {Sleep }}$ to save energy till the next Beacon arrives (see figure 3 .

Figure 4 shows the process of data exchange between EN participating in SR and the FC (AS). EN must send $D_{i}, l_{i}$ and $N_{s, i}$, and receives from the FC whether it is selected to perform SR in the next round and $N_{s, i}$ to be used.

Diagram of figure 5 resumes the steps to be followed in the proposed strategy for the participating and the not participating ENs in the network during one SR operation.

\footnotetext{
${ }^{3}$ In LoRa network, at each uplink operation, the EN uses channel parameters, i. e. SF and operating frequency, fixed by the NS in the previous operation and sent within Rx1.
} 


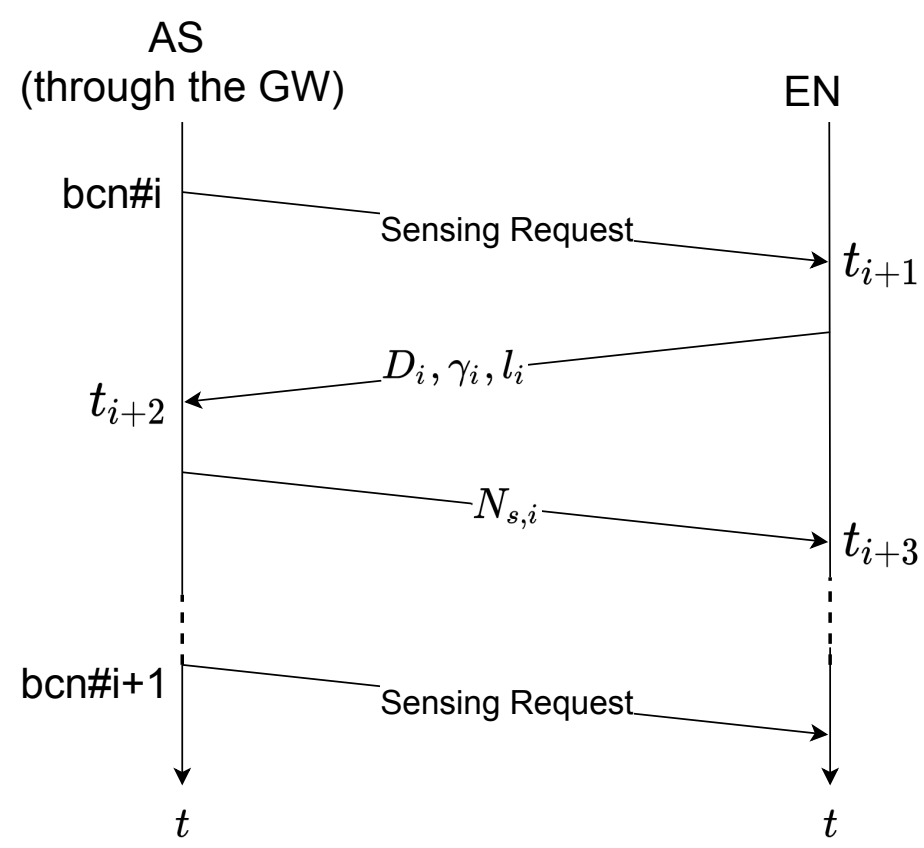

Figure 4: Our ESco strategy: After requesting the ENs to make SS, they respond to the AS request by $D_{i}, l_{i}$ and $\gamma_{i}$. AS makes a final decision on the PU channel availability and chooses the ENs with the $N_{E}$ highest ESs to establish SS in the next round. 


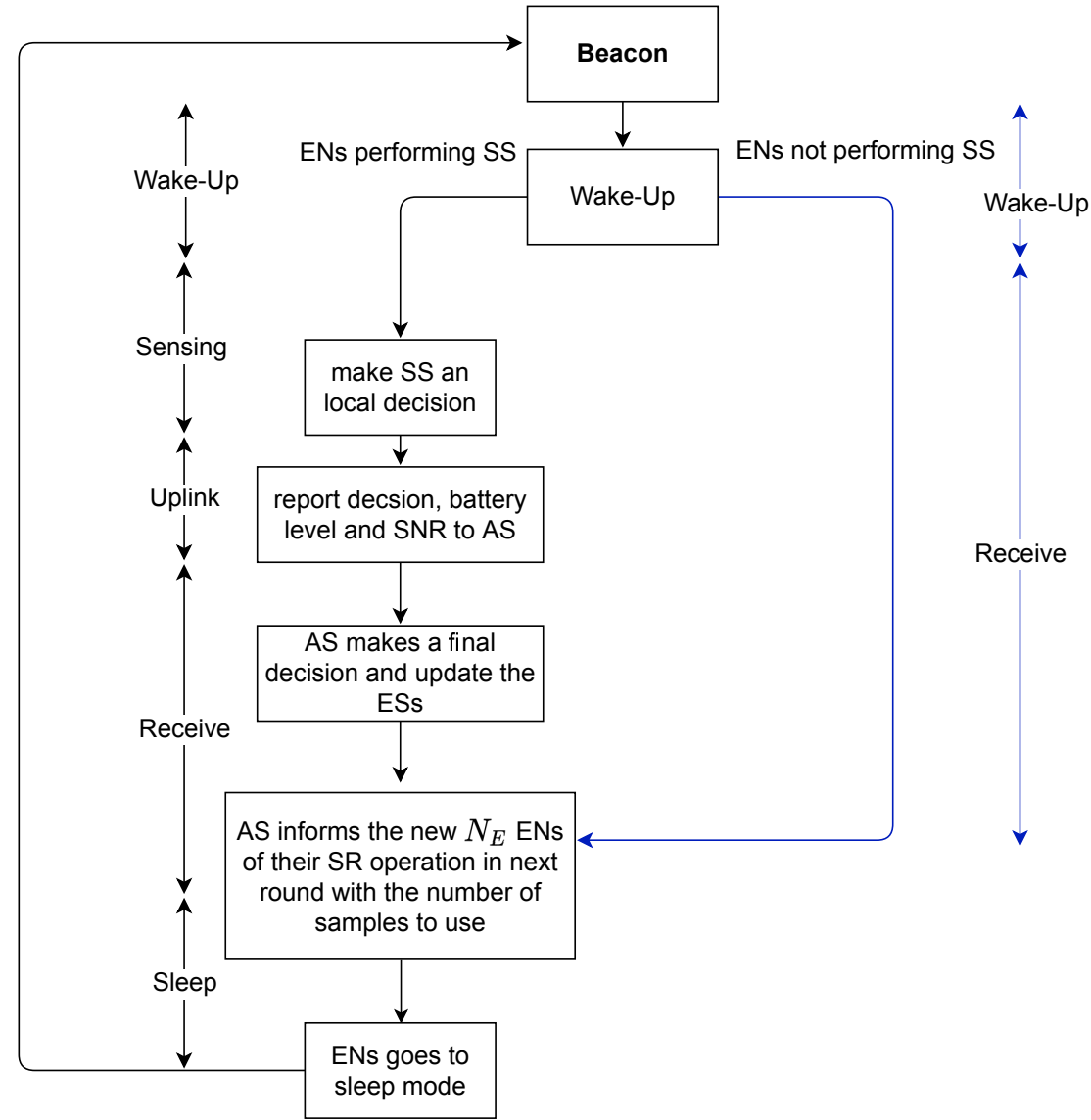

Figure 5: The behavior of the ENs during the SR operations: ENs wakes up for $T_{S R}$, then sleep till the next SR operation 


\subsection{Data Encoding of SNR and Battery Level}

For our application, the LoRaWAN packet is composed from the PayLoad (PL) and other bytes related to the communication identification between the EN and the AS. It must report to the AS three parameters: the decision on the channel $D_{i}$, the SNR $\gamma_{i}$ and the battery level $l_{i}$. Subsequently PL length depends on the number of bits on which $D_{i}, l_{i}$ and $\gamma_{i}$ measures are represented. Given that $D_{i}$ can be represented using one bit: 0 if PU is detected absent and 1 otherwise, the two other measures are not one-bit represented.

We assume that the battery level and SNR are quantized on $Q_{l}$ and $Q_{\gamma}$ levels respectively as follows:

$$
\begin{aligned}
Q_{l} & =\left[B_{0}, B_{1}, B_{2}, \ldots, B_{Q_{M_{l}-1}}\right] \\
Q_{\gamma} & =\left[0, \zeta_{1}, \zeta_{2}, \ldots, \zeta_{Q_{M_{g}}-1}\right]
\end{aligned}
$$

with: $Q_{\gamma}=2^{M_{l}}$ and $Q_{\gamma}=2^{M_{g}}$, where $M_{l}$ and $M_{g}$ are the bits number reserved to represent the battery levels and the SNR respectively. $B_{0}$ represents a battery level very close to zero, while $B_{Q_{l}}$ represents the full energy. $\zeta_{1}$ represents the minimum SNR, i.e. any SNR lower than $\zeta_{1}$ the SNR is considered null, and $\zeta_{Q_{M_{g}-1}}$ is the maximum SNR, where it represents the saturation level. The following equation summarizes the the quantization process of $\gamma_{i}$ :

$$
\gamma_{i} \rightarrow\left\{\begin{array}{l}
0 ; \text { if } \gamma_{i}<\zeta_{1} \\
\zeta_{k} ; \text { if } \zeta_{1} \leq \gamma_{i} \leq \zeta_{Q_{\gamma}} \\
\quad \text { and }\left\|\gamma_{i}-\zeta_{k}\right\|_{2} \leq\left\|\gamma_{i}-\zeta_{p}\right\|_{2}, k \neq p ; k, p \in\left[1, Q_{\gamma}\right] \\
\zeta_{Q_{\gamma}} ; \text { if } \gamma_{i}>\zeta_{Q_{M_{g}}-1}
\end{array}\right.
$$

Forcing SNR to be 0 as shown in the above equation makes the ES null, since $N_{s, i} \rightarrow$ as $\gamma_{i} \rightarrow 0$ (see equation (14)). This will help the CR-IoT network to avoid selecting ENs with very low SNR and prevents the SNR-wall phenomenon [23, which may happen when performing SS at low SNR values. In addition, low values of SNR highly consumes the battery since a huge number of samples is required to meet the detection requirement. Regarding the maximal value $\zeta_{Q_{\gamma}}$, this saturates the SNR value. In fact, at very high SNR, the EN could fulfil the detection requirement with a few number of samples. Thus, it positively impacts the amount of consumed energy by the SS operation.

\section{Simulation results}

In our simulations, we use real parameters related to the SS, the transmit/receive, and the Micro-Controller Unit (MCU). For the SS operation, the 330 parameters of RTL-SDR-based RadioHound are used [41]. It can scan from 25 $\mathrm{MHz}$ to $6 \mathrm{GHz}$, with a power less than $3 \mathrm{~W}$. For transmit/receive and MCU operations, we use respectively the parameters of transceiver SX1272 and the Semtech STM32L073 MCU [42, 43.

According to the experimental results of [44], the energy is mainly consumed 
335 by the four following functions of the EN: (1) The Measurement (Sensing of the channel in our application), (2) the transmission, (3) the Micro-Controller Unit (MCU) running, and (4) the receiving. Even though in Sleep mode the EN consumes a very low power, but it becomes considerable due to the long time taken by this mode. Table 2 gives the electrical specifications of the sys-

340 tem under the following parameters: Supply voltage $=3.3 \mathrm{~V}$, Capacity $=1000$ $\mathrm{mAh}$, Operating Frequency $=868 \mathrm{MHz}$, Power of transmission $=10 \mathrm{dBm}$, and the bandwidth (BW) of LoRa communication $=125 \mathrm{KHz}$.

Table 2: Power consumption specifications of the four main functions of the LoRa EN

\begin{tabular}{lll}
\hline Mode & Unit & Current \\
\hline Receive mode & SX1272 & $10.5 \mathrm{~mA}$ \\
\hline Transmit mode & SX1272 & $31 \mathrm{~mA}$ \\
\hline Spectrum Sensing & RadioHound & $0.9 \mathrm{~A}$ \\
\hline MCU Running & STM32L073 & $1.8 \mathrm{~mA}$ \\
\hline Sleep mode & SX1272 & $0.1 \mu \mathrm{A}$ \\
\hline
\end{tabular}

Receive Mode. In the receive mode, EN is active to hear from the AS two kinds of operations: (1) when receiving the Beacon from the GW considered as re345 quest to make the SS, the Beacon lasts for $16 \mathrm{~ms}$ [36. And (2), when receiving data in $R x 1$ informing the EN whether it is selected to make the SS in the next round or not. The time of $R x 1$ depends on the SF used by the EN in the transmission. $R x 2$ is used by the NS to send some updates to the ENs, and it is disregarded in this work.

Transmit Mode. The duration of the transmission operation is related directly to the number of transmitted symbols $N_{\text {symb }}$ :

$$
N_{\text {symb }}=8+\max [\mathcal{H}, 0]
$$

where $\mathcal{H}=\left\lceil\frac{28+8 P L+16 C R C-4 S F}{4(S F-2 D E)}\right\rceil(C R+4)$, where $\lceil X\rceil$ rounds $X$ to the nearest integer greater than or equal to $X$.

$P L$ represents number of bytes in the PL. CRC indicates the presence or not of the CRC field in the physical message (CRC is set to 0 if the CRC field is not present; otherwise, CRC is equal to 1 ). CR denotes the coding rate and can take values from 1 to 4 , for $4 / 5$ to $4 / 8$ coding rate, respectively. Finally, $D E=1$ when the low data rate optimization is enabled (for $\mathrm{SF}=12$ and $\mathrm{SF}=11$ ) and $D E=0$ elsewhere.

The transmission time $T_{T x}$ is evaluated using the duration $T_{s y m b}$ of the $N_{s y m b}$ symbols and the preamble message time $T_{\text {pre }}$ (refer to [38, 44] for more infor- 
mation), where:

$$
\begin{aligned}
T_{\text {symb }} & =N_{\text {symb }} \times \frac{2^{S F}}{B W} \\
T_{\text {pre }} & =\left(N_{\text {pre }}+4.5\right) \times \frac{2^{S F}}{B W} \\
T_{T x} & =T_{\text {symb }}+T_{\text {pre }}
\end{aligned}
$$

$N_{\text {pre }}$ is the number of preamble symbols, and it is fixed in this study to 8 .

Spectrum Sensing Mode. The energy consumed by SS depends on the number of samples needed for the energy detector before making the decision. We assume that the band to be monitored is $125 \mathrm{KHz} \mathrm{BW}$, and the sampling frequency $f_{s}$ is twice the BW. Thus the sensing time $T_{S S}$ at the $\mathrm{EN}_{i}$ becomes:

$$
T_{S S}=\frac{N_{s, i}}{f_{s}}
$$

355 Even though the power of SS is relatively high compared to other modes, the time of SS is very short, which leads to a comparable consumed energy to other modes.

Micro-Controller Unit Running Mode. This mode is activated once the system wakes up and lasts till the end of the receiving mode.

Sleep Mode. After receiving the data from the NS and AS in $R x 1$, the EN system goes to Sleep mode till the time of the next SS round.

\subsection{Simulation scenarios}

ESco is compared to two other strategies: (1): $N_{E}$ ENs are randomly selected to make the SS, and (2) ENs with the $N_{E}$ maximal energy level make

For the three strategies, the network is considered dead when the last number of ENs with $l_{i}>0$ is smaller than $N_{E}$. The total number of nodes in the networks is fixed to $N_{t}=500$ throughout the upcoming simulation results.

The ENs are assumed to be equipped with the same battery capacity, and $l_{i}$ is tions. The battery lifetime is considered to evaluate the proposed mechanism. This lifetime is related to the initial energy of the battery as well as the adopted $\mathrm{SF}$ and the SNR at the node. High SF means that the transmission time becomes large, and subsequently the consumed energy increases compared to the 375 scenario when low SF is used. On the other hand, low SNR leads to the need of a large number of samples to make an accurate SS, which increases the consumed energy of the SS as shown in equation 22 .

Regarding the parameters of SS, the target $\overline{P_{d}}$ and $\overline{P_{f a}}$ are fixed to 0.9 and 0.1 respectively. The SNR is assumed to be log-normal distributed among the ENs, 


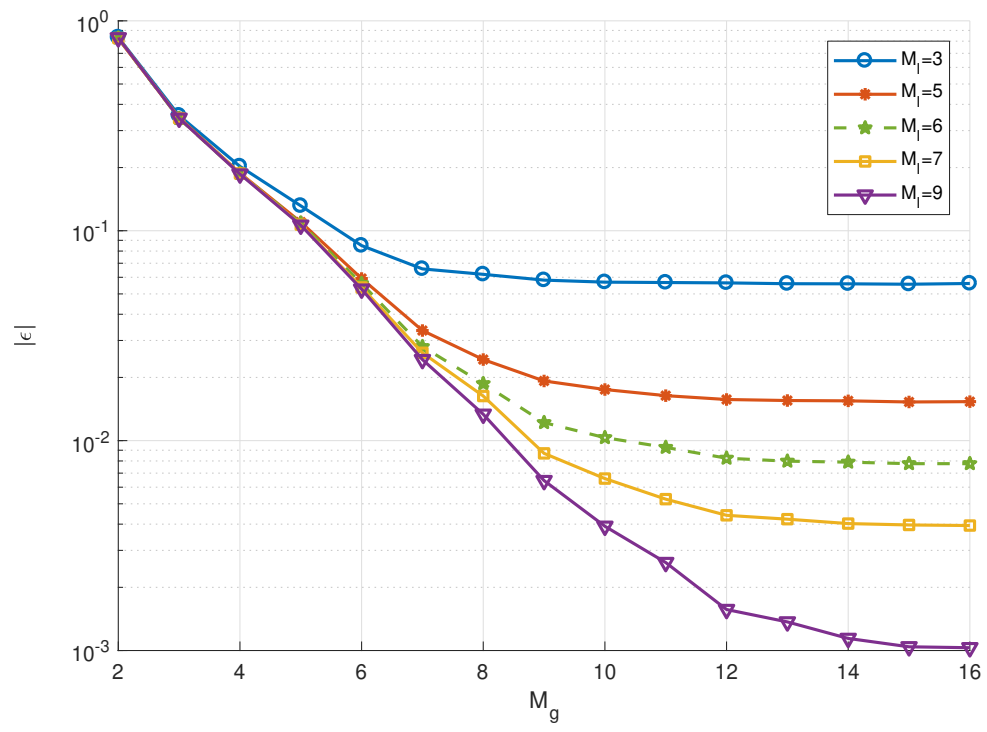

Figure 6: Variation of the error of quantization $\epsilon$ is terms of the number of bits used in $Q_{\gamma}$ and $Q_{l}$.

\subsection{Investigation on the size of $P L$}

The precision of the quantization of SNR and the battery level is necessary to make a reliable decision at the AS. From one hand, the quantization error may adversely affect the ES, leading to wrong selection of the ENs. On the other hand, $N_{s, i}$ of ENs participating in the SR is calculated at the AS based on the quantized SNR for target $\overline{P_{d}}$ and $\overline{P_{f a}}$. If the SNR's quantization is badly done, the EN may not meet the detection requirements since $N_{s, i}$ becomes irrelevant to the real SS conditions of the EN. Given that $D_{i}$ is represented on one bit, we aim at investigating the number of bytes to be loaded by the PL in the transmitted packet. $\gamma_{i}$ is log-normal distributed with $\gamma_{m}=-10$ and $\sigma_{m}=\sqrt{10}$ $\mathrm{dB}$. The minimum SNR is set to $-20 \mathrm{~dB}$. The maximal value of SNR is set to $5 \mathrm{~dB}$, beyond this value the SNR is saturated as per the model of (17).

The evaluation of the reliability of the quantization is based on the error $\epsilon$ :

$$
\epsilon=\frac{E\left[\left|\mathcal{F}\left(l_{i}, N_{s, i}\right)-\mathcal{F}_{q}\left(l_{i}, N_{s, i}\right)\right|\right]}{E\left[\mathcal{F}\left(l_{i}, N_{s, i}\right)\right]}
$$

where $\mathcal{F}_{q}\left(l_{i}, N_{s, i}\right)$ is the ES evaluated based on the quantized $\gamma_{i}$ and $N_{s, i}$, and $E[\cdot]$ stands for the mathematical expectation.

Figure 6 presents the evolution of $\epsilon$ for several values of $Q_{\gamma}$ and $Q_{l}$. As expected, $385 \epsilon$ decreases with the increase of $M_{g}$ and $M_{l}$. For $M_{l}=9$ bits and $M_{g} \geq 9$ the error becomes lower than $1 \%$. At $M_{l}=9$ bits and $M_{g}=12$, the error becomes close to $0.1 \%$.

Throughout the rest of the paper $M_{l}=9$ and $M_{g}=12$ are fixed to evaluate the performance of the proposed strategy ESco. 


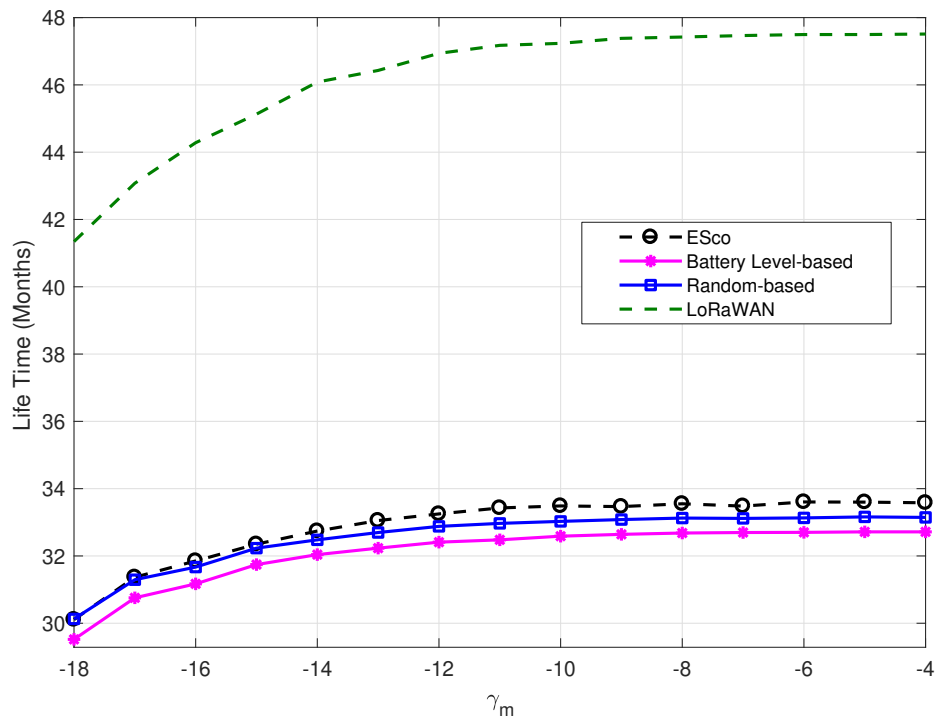

Figure 7: The lifetime of the network in terms of the mean SNR $\gamma_{m} . P_{f a}=0.1 ; P_{d}=0.9 ; N_{t}=$ 500

\subsection{Lifetime and sensing performance evaluation}

In this subsection, we analyse the performance of the CR-IoT from the sensing and the lifetime points of view.

Due to the modifications on the LoRaWAN protocol, especially the need of all the ENs to be awake at the beginning of each transmission period, the energy efficiency may be changed. Figure 7 shows the network lifetime for several values of $\gamma_{m}$ while comparing with the standard LoRaWAN, where not all the ENs wake up at the beginning of the period. $\sigma_{g}$ is fixed to $\sqrt{10} \mathrm{~dB}$ and the number of nodes participating in each SR operation is fixed to $N_{E}=8$. The lifetime of the network increases with $\gamma_{m}$ for all the presented strategies since the number of samples to be treated decreases. Thus, the energy spent by the SS operation decreases. On the other hand, the proposed ESco outperforms both Random-based and Battery level-based strategies by achieving highest lifetime. The variation of $\gamma_{m}$ from $-18 \mathrm{~dB}$ to $-4 \mathrm{~dB}$ leads to increase the lifetime of 405 ESco by more than three months and a half, and about three months for both Random-based and Battery level-based strategies. Compared to LoRaWAN, the lifetime of the ENs adopting ESco exhibits a decrease of $25 \%$, from 47 months at $\gamma_{m}=-14$ to around 33 months for the same SNR. This is due to the fact of the waking up of all the ENs at the beginning transmission period. This paid cost comes at providing the LoRaWAN with the ability to apply on-request data transmission which is not available for the actual LoRaWAN protocol, and, on the other hand, to make the LoRa network work cooperatively to select the ENs with the best ESs. 


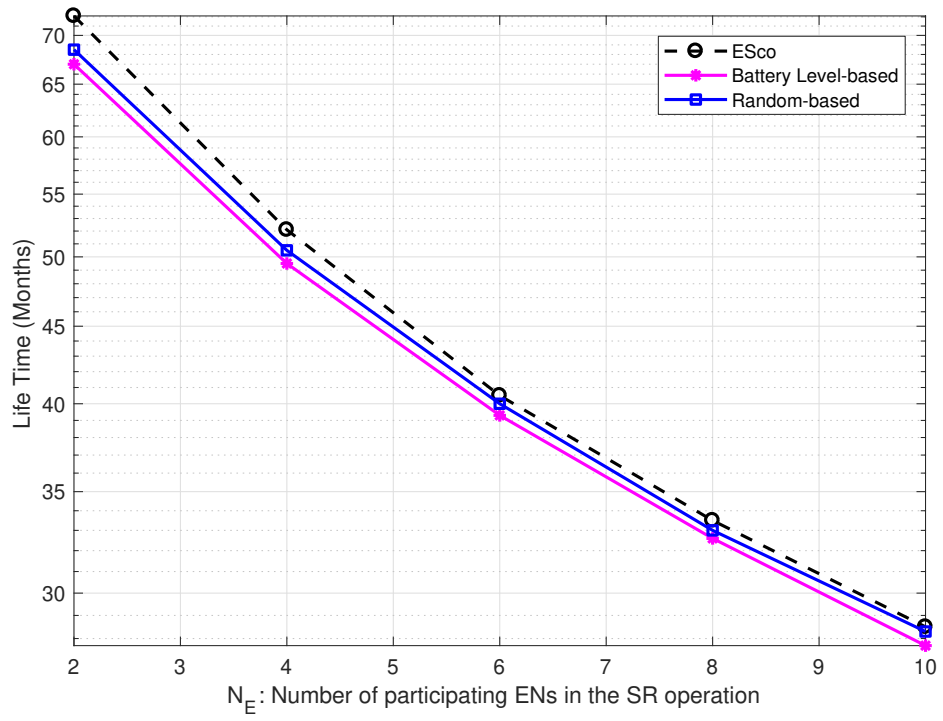

Figure 8: The impact of $N_{E}$ on the network lifetime when $P_{f a}=0.1 ; P_{d}=0.9 ; N_{t}=500$, and $\mathrm{SF}=9$.

The chosen number of the participating ENs, $N_{E}$, may play an essential role in the SR process since this parameter is related to the spatial diversity to be ensured by the cooperative SS. Figure 8 shows the evolution of the network lifetime in terms of $N_{E}$. The lifetime decreases as $N_{E}$ increases. However, for all the considered values of $N_{E}$ our proposed strategy reaches the longest lifetime compared to other considered strategies. Even though the sensing time decreases with $N_{E}$, leading to reduce the consumed energy of SS, the lifetime is decreasing with $N_{E}$ because the participating ENs consumes a high transmission energy to report the sensing results to the AS. However, a sufficiently high number of the ENs is required in order to ensure the spatial diversity that overcomes the problem of the hidden PU problem [22].

Figure 9 shows the impact of the adopted SF on the lifetime of the network. SF varies form 7 to 12 according to the specifications of LoRaWAN. The lifetime decreases with the increase of SF for the three presented strategies. This refers to the fact that the transmission time increases with SF, which leads to increase the transmission energy. However, the proposed ESco outperforms the two other strategies for all the used SFs.

Figure 10 depicts the impact of $N_{t}$ on the lifetime of the network. $N_{E}=8$ is adopted. As $N_{E}$ increases the network lifetime increases. This is because the probability of an EN is selected to perform the SS decreases with $N_{t}$ for all the presented strategies. This leads to extend the lifetime of the sensor and subsequently the one of the network. Our proposed ESco preserves better the lifetime of the network compared to the two other strategies as show in figure 10. 


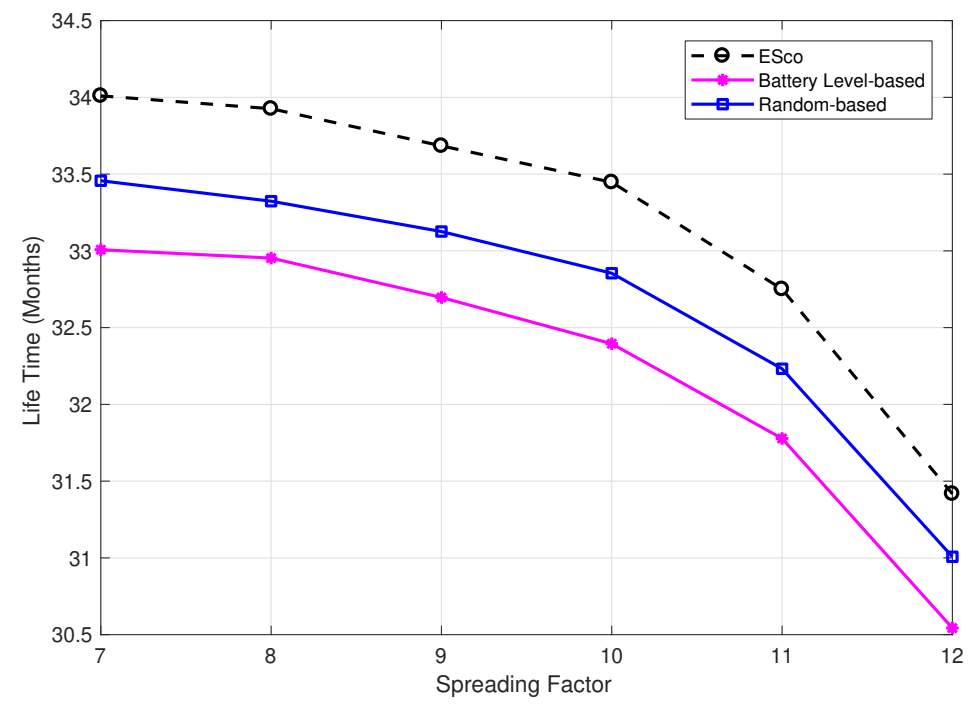

Figure 9: The evolution of the network lifetime with respect to SF. $N_{E}=8, N_{t}=500$

One of the essential parameters to be set in any CR-based network is the time between two consecutive SS operations. This is related to the rate of a tolerable collision time between $\mathrm{SU}$ and $\mathrm{PU}$, and this collision rate may differ from one primary channel to another. In the proposed protocol, AS should request a SS operation each $T_{b c n}$ using a Beacon, where the request rate may highly impact the lifetime of the ENs. Figure 11 shows the evolution of the 445 lifetime of the network for several of $T_{b c n}$. As depicted, the lifetime is highly impacted by $T_{b c n}$ since at each SR operation the EN must spend energy for sensing, receiving and reporting. For ESco, which outperforms the two other strategies, achieves a lifetime of 34 months for $T_{b c n}=128 \mathrm{sec}$, while this lifetime is reduced to 18 months when $T_{b c n}=32 \mathrm{sec}$. The lifetime becomes almost the double when using $T_{b c n}=128$ instead of $T_{b c n}=32$. Thus, increasing $T_{b c n}$ the lifetime of the network increases; however, this may increase the collision risque with the PU since the latter may return active between two consecutive Beacons. Further discussion on the collision is given in the next subsection.

\subsection{Throughput and Collision Analysis}

In this section, we analyse the impact of the length of $T_{b c n}$ on the throughput and the collision rate between the secondary transmission and PU. Secondary transmission is based on the decision made by the CR-IoT. Useful throughput $R_{0}$ of the secondary transmission is done without any interference with PU, while the colliding throughput $R_{1}$ is considered when $\mathrm{SU}$ sends the data with an active PU on the same channel. $R_{0}$ happens when the CR-IoT makes a true decision on the absence of PU and the latter does not returns back during $T_{b c n}$. $R_{1}$ takes place is two cases: (1) if the CR-IoT makes a miss-detection on PU 


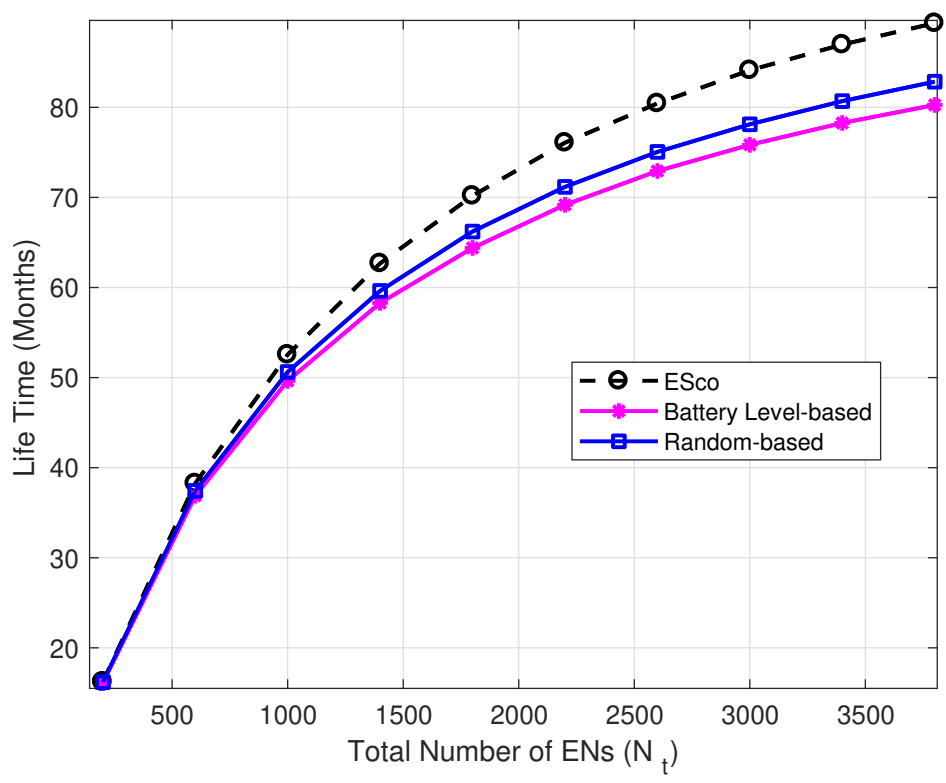

Figure 10: The impact of $N_{t}$ on the network lifetime when $N_{E}=8$.

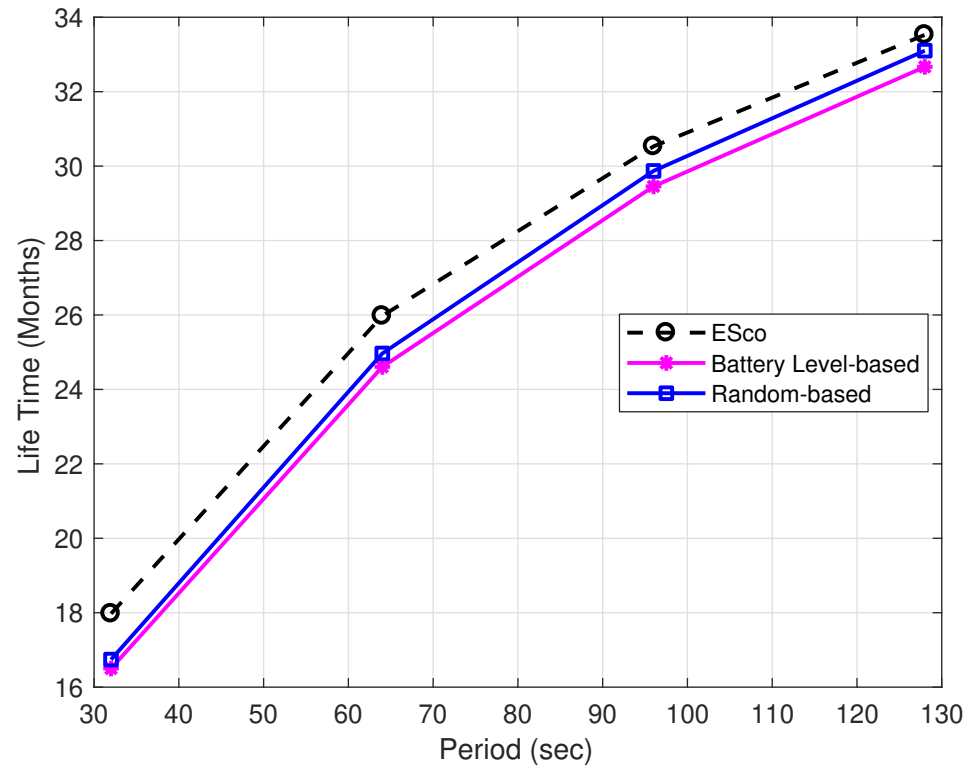

Figure 11: The impact of the length of Beacon period on the network lifetime with $N_{E}=8$. 
presence, and (2) CR-IoT makes a true decision on the absence of PU when doing the SS, but PU returns back during $T_{b c n}$. Given that the PU can change its state (active to idle or idle to active) once during $T_{b c n}$, the probability of collision $P_{c}$ and collision-free $P_{c f}$ are given as follows [39, 45]:

$$
\begin{aligned}
P_{c} & =\left(1-P_{d}\right) p_{1}+\left(1-P_{f a}\right) p_{0} P_{r} \\
P_{c f} & =\left(1-P_{f a}\right) p_{0}\left(1-P_{r}\right)
\end{aligned}
$$

where $p_{0}$ and $p_{1}$ are the probabilities that $\mathrm{PU}$ is absent and active respectively when the SS is performed. $P_{r}$ is the probability that PU reappears during $T_{b c n}-T_{S S}$, and it is given by:

$$
P_{r}=1-\exp \left(-\frac{T_{b c n}-T_{S}}{\lambda}\right)
$$

where $\lambda$ is defined as the average idle duration of the PU.

Subsequently, $R_{0}$ and $R_{1}$ are given is the following equations:

$$
\begin{aligned}
& R_{0}=C_{0} P_{c f} \\
& R_{1}=C_{1} P_{c}
\end{aligned}
$$

$C_{0}$ and $C_{1}$ are the channel capacities related to $R_{0}$ and $R_{1}$ respectively:

$$
\begin{aligned}
& C_{0}=W \log _{2}\left(1+Z_{0}\right) \\
& C_{1}=W \log _{2}\left(1+Z_{1}\right)
\end{aligned}
$$

where $W$ is the transmission bandwidth (in our case $W=125 \mathrm{kHz}$ ), $Z_{0}$ and $Z_{1}$ are the SNR and the Signal to Noise and Interference Ratio (SNIR) of the secondary transmitted signal at the secondary receiver respectively.

Figure 12 presents the evolution of the collision probability, collision-free probability, and the throughputs of the secondary system. $Z_{0}=5 \mathrm{~dB}$ and $Z_{1}=3$ ${ }_{460} \mathrm{~dB}$ are fixed. $\lambda=130 \mathrm{sec}, p_{0}=0.3$ and $p_{1}=0.7$ are adopted. As shown in figure 12(a), $P_{c}$ increases with $T_{b c n}$ from 0.08 at $T_{b c n}=32 \mathrm{sec}$ to greater than 0.18 at $T_{b c n}=128 \mathrm{sec}$. This reflects the adverse impact of a long $T_{b c n}$ on the collision between the secondary and the primary transmissions. On the other hand, $P_{c f}$ decreases with $T_{b c n}$. Thus, low $T_{b c n}$ reduces the secondary collision to the primary transmission while enhances the collision-free activity of the secondary system.

Figure 12(b) depicts the evolution of both the useful and the colliding throughputs of the secondary transmission. $R_{0}$ decreases with $T_{\text {beacon }}$ while $R_{1}$ increases. In fact, a long $T_{b c n}$ may reduce the frequency efficiency of the sec470 ondary system, since it takes a long time to re-establish a SS after a decision of the presence of PU. During this time, PU may leave the channel, thus a lost in exploiting the available frequency resources happens. In turns, the long time may also increase the collision probability since PU may return active between two consecutive SS operation, when the secondary system is unaware of PU ${ }_{475}$ activity. Thus shortening this time leads to reduce the collision rate. 


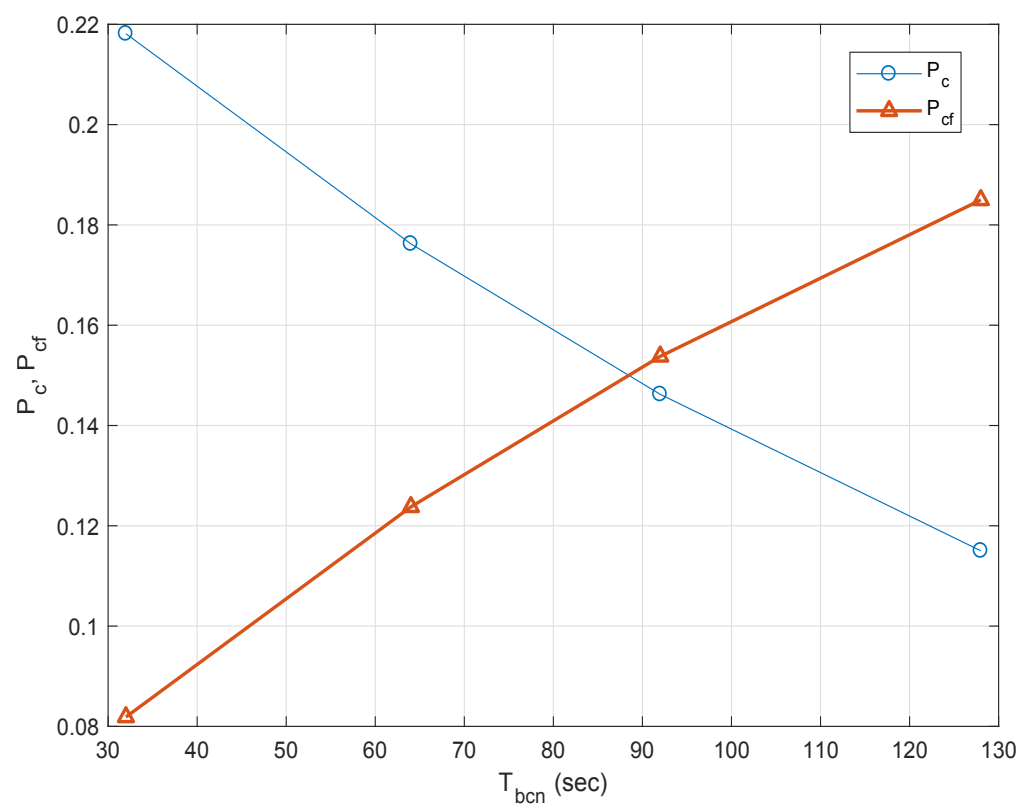

(a) The probability of Collision

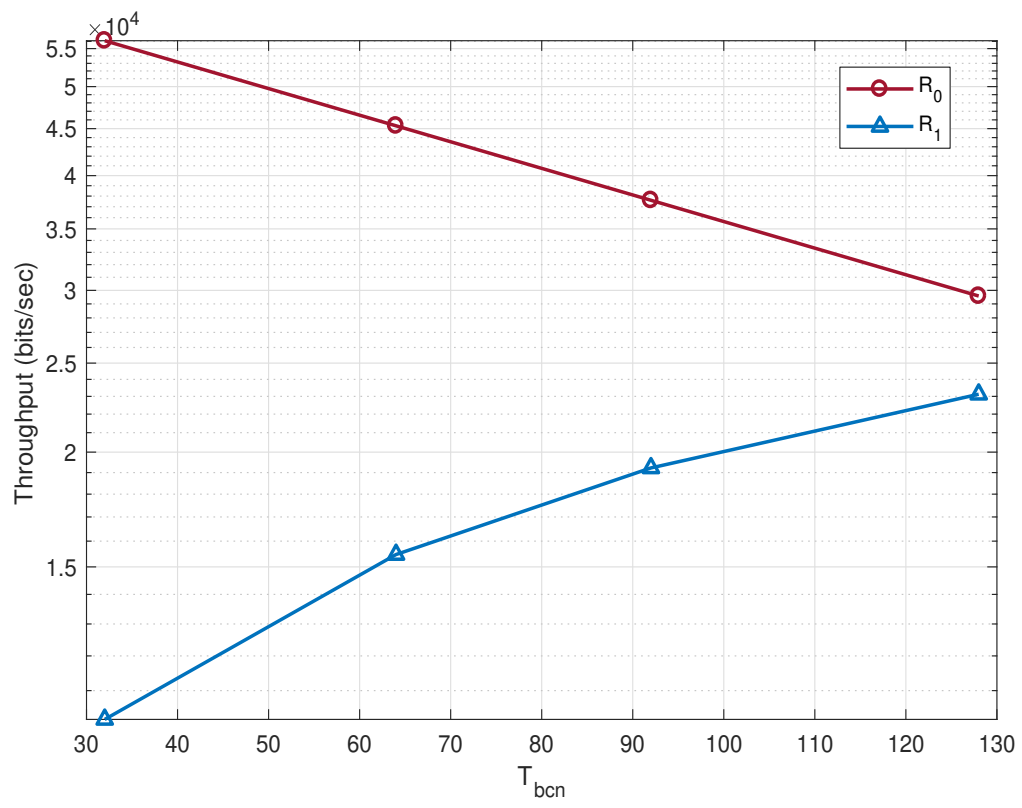

(b) The Throughput

Figure 12: The evolution of the collision and collision-free probability, and the useful and colliding throughputs in terms of $T_{b c n}$ 


\section{Conclusion}

In this paper, Eligibility Score-based (ESco) strategy is proposed to handle the sensing-reporting process in a battery-powered CR-IoT network providing Spectrum Sensing as a Service. LoRaWAN protocol is taken as case study to implement the proposed strategy. The SS operation performed in such network may largely consume the battery of the ENs. Thus the ENs involved in the SS should be carefully selected so that the lifetime of the CR-IoT network is extended as possible. The selection process is done based on an efficient Eligibility Score (ES) evaluated at each EN in the network. This ES takes into consideration both SS decision reliability and the battery level of the EN. In addition, we studied the data exchange between Network Server and EN of proposed ESco as per the LoRaWAN Class-B protocol, where we introduce needed modifications to make this protocol responsive to the on-demand model of the Spectrum Sensing as a Service. Numerical results depict the efficiency of the 490 proposed strategy by respecting high protection of PU against the interference, high spectrum efficiency and long extended network lifetime. The compatibility of ESco with other IoT technologies such as Sigfox and NBIoT are to be explored and investigated in future work.

\section{References}

[1] Statistica, Internet of Things - number of connected devices worldwide 2015-2025, available at https://www.statista.com/statistics/ 471264/iot-number-of-connected-devices-worldwide/ (Statstica 2020 (accessed March 07, 2020)).

[2] S. H. Shah, I. Yaqoob, A survey: Internet of Things (IOT) technologies, applications and challenges, in: 2016 IEEE Smart Energy Grid Engineering (SEGE), 2016, pp. 381-385. doi:10.1109/SEGE.2016.7589556.

[3] L. Atzori, A. Iera, G. Morabito, The Internet of Things: A survey, Computer Networks 54 (15) (2010) 2787 - 2805. doi:https: //doi.org/10.1016/j.comnet.2010.05.010. URL http://www.sciencedirect.com/science/article/pii/ S1389128610001568

[4] A. A. Khan, M. H. Rehmani, A. Rachedi, Cognitive-Radio-Based Internet of Things: Applications, Architectures, Spectrum Related Functionalities, and Future Research Directions, IEEE Wireless Communications 24 (3) (2017) 17-25. doi:10.1109/MWC.2017.1600404.

[5] H. Tabassum, A. H. Sakr, E. Hossain, Analysis of Massive MIMO-Enabled Downlink Wireless Backhauling for Full-Duplex Small Cells, IEEE Transactions on Communications 64 (6) (2016) 2354-2369. 
535

[6] T. Yucek, H. Arslan, A Survey of Spectrum Sensing Algorithms for Cognitive Radio Applications, IEEE Communication Surveys \& Tutorials 11 (1) (2009) $116-130$.

[7] M. Wyglinski, M. Nekovee, Y. Hou, Cognitive Radio Communications and Networks, Principles and Practice, Elsevier, 2010.

[8] B. Wang, K. J. R. Liu, Advances in cognitive radio networks: A survey, IEEE Journal of Selected Topics in Signal Processing 5 (1) (2011) 5-23.

[9] B. Di Martino, M. Rak, M. Ficco, A. Esposito, S. Maisto, S. Nacchia, Internet of things reference architectures, security and interoperability: A survey, Internet of Things 1 (2018) 99-112.

[10] S. Bayhan, A. Zubow, A. Wolisz, Spass: Spectrum sensing as a service via smart contracts, in: 2018 IEEE International Symposium on Dynamic Spectrum Access Networks (DySPAN), IEEE, 2018, pp. 1-10.

[11] C. Perera, A. Zaslavsky, P. Christen, D. Georgakopoulos, Sensing as a service model for smart cities supported by internet of things, Transactions on emerging telecommunications technologies 25 (1) (2014) 81-93.

[12] W. Ejaz, G. A. Shah, N. u. Hasan, H. S. Kim, Energy and throughput efficient cooperative spectrum sensing in cognitive radio sensor networks, Transactions on Emerging Telecommunications Technologies 26 (7) (2015) 1019-1030. arXiv:https://onlinelibrary.wiley.com/doi/pdf/ 10.1002/ett.2803, doi:10.1002/ett.2803 URL https://onlinelibrary.wiley.com/doi/abs/10.1002/ett.2803

[13] A. A. Khan, M. H. Rehmani, A. Rachedi, When Cognitive Radio meets the Internet of Things?, in: 2016 International Wireless Communications and Mobile Computing Conference (IWCMC), 2016, pp. 469-474. doi: 10.1109/IWCMC.2016.7577103.

[14] P. Rawat, K. D. Singh, J. M. Bonnin, Cognitive radio for M2M and Internet of Things: A survey, Computer Communications 94 (2016) 1 29. doi:https://doi.org/10.1016/j.comcom.2016.07.012.

(1) URL http://www.sciencedirect.com/science/article/pii/ S0140366416302699

[15] A. Aijaz, A. H. Aghvami, Cognitive Machine-to-Machine Communications for Internet-of-Things: A Protocol Stack Perspective, IEEE Internet of Things Journal 2 (2) (2015) 103-112. doi:10.1109/JIOT.2015.2390775.

[16] B. Moon, Dynamic spectrum access for internet of things service in cognitive radio-enabled lpwans, Sensors 17 (12). doi:10.3390/s17122818 URL https ://www.mdpi.com/1424-8220/17/12/2818 
[17] W. Ejaz, M. Ibnkahla, Multiband Spectrum Sensing and Resource Allocation for IoT in Cognitive 5G Networks, IEEE Internet of Things Journal 5 (1) (2018) 150-163. doi:10.1109/JIOT.2017.2775959.

[18] M. B. H. Weiss, S. Delaere, W. H. Lehr, Sensing as a service: An exploration into practical implementations of dsa, in: 2010 IEEE Symposium on New

[19] O. Grøndalen, M. Lähteenoja, P. Grønsund, Evaluation of business cases for a cognitive radio network based on wireless sensor network, in: 2011

[27] A. Nasser, A. Mansour, K.-C. Yao, H. Charara, M. Chaitou, Spectrum sensing for full-duplex cognitive radio systems, in: International Conference on Cognitive Radio Oriented Wireless Networks, Springer, 2016, pp. 363374 . 
37 Mroue, B. Parrein, S. Hamrioui, P. Bakowski, A. Nasser, E. M. n Cruz, W. Vince, LoRa+: An extension of LoRaWAN protocol to reduce 620 infrastructure costs by improving the Quality of Service, Internet of Things 9 (2020) 100176. doi:https://doi.org/10.1016/j.iot.2020.100176. URL S2542660520300160

[38] L. Casals, B. Mir, R. Vidal, C. Gomez, Modeling the energy performance 625

[29] A. Ghasemi, E. Sousa, Collaborative spectrum sensing for opportunistic access in fading environments, Proceeding of IEEE DySPAN (2004) 131136.

[30] S. wen WU, J. kang ZHU, L. QIU, M. ZHAO, Snr-based weighted cooperative spectrum sensing in cognitive radio networks, The Journal of China Universities of Posts and Telecommunications 17 (2) (2010) 1 - 7. doi:https://doi.org/10.1016/S1005-8885(09)60437-4. URL http://www.sciencedirect.com/science/article/pii/ S1005888509604374

[31] Y. Selen, H. Tullberg, J. Kronander, Sensor selection for cooperative spectrum sensing, in: 2008 3rd IEEE Symposium on New Frontiers in Dynamic Spectrum Access Networks, 2008, pp. 1-11.

[32] A. S. Cacciapuoti, I. F. Akyildiz, L. Paura, Correlation-aware user selection for cooperative spectrum sensing in cognitive radio ad hoc networks, IEEE Journal on Selected Areas in Communications 30 (2) (2012) 297-306.

[33] C. Gu, L. Jiang, R. Tan, Lora-based localization: Opportunities and challenges, arXiv preprint arXiv:1812.11481.

[34] D. Bankov, E. Khorov, A. Lyakhov, Lorawan modeling and mcs allocation to satisfy heterogeneous qos requirements, Sensors 19 (19). doi:10.3390/ s19194204. URL https ://www .mdpi.com/1424-8220/19/19/4204

[35] A. Farhad, D.-H. Kim, J.-Y. Pyun, Resource allocation to massive internet of things in lorawans, Sensors 20 (9). doi:10.3390/s20092645 URL https://www.mdpi.com/1424-8220/20/9/2645

36] LoRaWAN ${ }^{\mathrm{TM}}$ 1.0.3 Specification, Notice of Use and Disclosure, available at https://lora-alliance.org/sites/default/files/2018-06/ lorawan1.0.3_final_0.pdf (2018). of lorawan, Sensors 17 (10). doi:10.3390/s17102364. URL https://www.mdpi.com/1424-8220/17/10/2364 
[39] Y. C. Liang, Y. Zeng, E. C. Y. Peh, A. T. Hoang, Sensing-Throughput Tradeoff for Cognitive Radio Networks, IEEE Transactions on Wireless Communications 7 (4) (2008) 1326-1337.

[40] Y. Liao, T. Wang, L. Song, Z. Han, Listen-and-Talk: Protocol Design and Analysis for Full-Duplex Cognitive Radio Networks, IEEE Transactions on Vehicular Technology 66 (1) (2017) 656-667.

[41] N. Kleber, A. Termos, G. Martinez, J. Merritt, B. Hochwald, J. Chisum, A. Striegel, J. N. Laneman, Radiohound: A pervasive sensing platform for sub-6 ghz dynamic spectrum monitoring, in: 2017 IEEE International Symposium on Dynamic Spectrum Access Networks (DySPAN), 2017, pp. $1-2$.

[42] S. Documen, SX1272 Development Kit User Guide, available at https: //www.semtech.com/uploads/documents/sx1272ska_userguide.pdf. (2018).

[43] S. M. Document, STM32L073x8 STM32L073xB STM32L073xZ Data Sheet, available at https://www.st.com/resource/en/datasheet/ stm321073v8.pdf (2018).

[44] T. Bouguera, J.-F. Diouris, J.-J. Chaillout, R. Jaouadi, G. Andrieux, Energy consumption model for sensor nodes based on lora and lorawan, Sensors $18(7)$. doi:10.3390/s18072104. URL https://www .mdpi.com/1424-8220/18/7/2104

[45] V. Towhidlou, M. Shikh-Bahaei, Adaptive full-duplex communications in cognitive radio networks, IEEE Transactions on Vehicular Technology 67 (9) (2018) 8386-8395. 